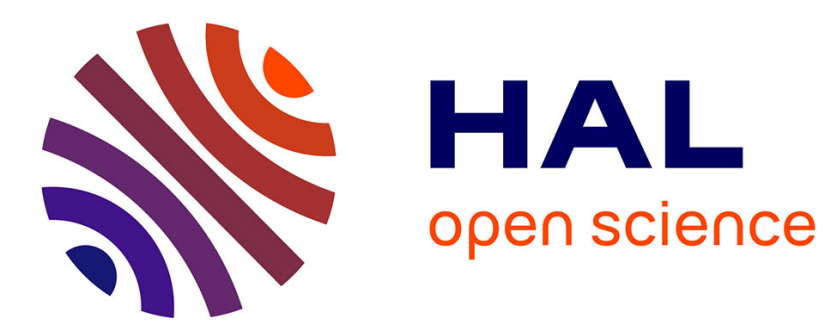

\title{
Natural vibrations in a system of nanotubes
}

Victor A. Eremeyev, Elena A. Ivanova, Nikita F. Morozov, S. E. Strochkov

\section{To cite this version:}

Victor A. Eremeyev, Elena A. Ivanova, Nikita F. Morozov, S. E. Strochkov. Natural vibrations in a system of nanotubes. Journal of Applied Mechanics and Technical Physics, 2008, 49 (2), pp.291-300. hal-00829633

\section{HAL Id: hal-00829633 \\ https://hal.science/hal-00829633}

Submitted on 3 Jun 2013

HAL is a multi-disciplinary open access archive for the deposit and dissemination of scientific research documents, whether they are published or not. The documents may come from teaching and research institutions in France or abroad, or from public or private research centers.
L'archive ouverte pluridisciplinaire $\mathbf{H A L}$, est destinée au dépôt et à la diffusion de documents scientifiques de niveau recherche, publiés ou non, émanant des établissements d'enseignement et de recherche français ou étrangers, des laboratoires publics ou privés. 


\title{
NATURAL VIBRATIONS IN A SYSTEM OF NANOTUBES
}

\author{
V. A. Eremeyev, ${ }^{1,4}$ E. A. Ivanova, ${ }^{2}$ \\ N. F. Morozov, ${ }^{3}$ and S. E. Strochkov ${ }^{4}$
}

Natural vibrations in a system of parallel micro- and nanotubes attached horizontally to an elastic substrate are analyzed. It is shown that several first eigenfrequencies corresponding to flexural vibrations of a single nanotube can be identified with the use of the linear shell theory within the frequency spectrum of an "integrated system" consisting of a substrate and nanotubes. This allows the flexural rigidity of a single nanotube to be evaluated. The resultant conclusion is supported by finite-element modeling based on the three-dimensional theory of electroelasticity. Results of a modal analysis of gallium arsenide nanotubes are presented.

Key words: nanomechanics, natural vibrations, nanotubes, flexural rigidity, shells, electroelasticity, finite element method.

A key problem in nanomechanics is determining mechanical and physical features of nanoobjects. Nanoobjects are known to have anomalous properties, which offer much promise in applications and do not depend, generally speaking, on characteristics of macroscopic samples [1-5]. An effective method for determining the moduli of elasticity, in particular, the flexural rigidity used in mechanics of macroobjects, is based on measuring the eigenfrequencies of such objects. Application of this method to nanoobjects involves the problem of measuring their eigenfrequencies. Such measurements are difficult to perform with the use of optical methods [6]. The essence of these methods is as follows. The examined object mounted on a macroscopic frame is excited with a laser beam; a second laser beam is then used to register the amplitude of vibrations at a certain point of the object, the signal is converted into an electrical signal, and the spectrogram obtained is analyzed with the help of a spectrometer, which finally yields the eigenfrequencies of the object. An important but not the only factor that restricts the area of applicability of the method is the fact that the laser beam is a spot with a diameter of the order of the light wavelength rather than a point. That is why the measured data are meaningless if the object diameter is smaller than the laser-beam diameter. Thus, the optical methods described above do not permit the frequency of isolated nanoobjects to be measured. Simultaneously, it is fairly possible to measure the eigenfrequencies of a system consisting of a microsubstrate and a regular array of identical nanoobjects. The main problem arising in measuring the frequencies of objects attached to an elastic substrate is the redistribution of eigenfrequencies in the object-substrate system between the eigenfrequencies of the object and the substrate individually [7]. This phenomenon is well known in mechanics.

Previously $[8,9]$ we proposed a method for determining the eigenfrequencies of several types of nanostructures (nanotubes and nanocrystals) on the basis of the measured eigenfrequencies of an "integrated system" consisting of a highly ordered array of identical nanotubes or nanocrystals grown on the substrate and protruding in the normal-tosubstrate direction. Nanocrystal arrays examined in [8,9] are exemplified with zinc oxide semiconductor micro- and nanocrystals. Such crystals possess good mechanical and physical properties presenting interest in nanomechanics

${ }^{1}$ Southern Scientific Center, Russian Academy of Sciences, Rostov-on-Don 344006; eremeyev@math.rsu.ru. ${ }^{2}$ St. Petersburg State Polytechnical University, St. Petersburg 195251; ivanova@EI5063.spb.edu. ${ }^{3}$ St. Petersburg State University, St. Petersburg 198904; morozov@NM1016.spb.edu. 'Southern Federal University, Rostov-onDon 344090; s-sterg@yandex.ru. Translated from Prikladnaya Mekhanika i Tekhnicheskaya. Fizika, Vol. 49, No. 2, pp. 160-171, March-April, 2008. Original article submitted March 26, 2007 
and nanophotonics. They can be obtained by various techniques, in particular, by pulsed laser deposition [10-12]. Usually, the sizes of nanoobjects in the array are roughly identical, which makes it possible to use the macroscopic size of the array to study nanoobject properties through determining several first eigenfrequencies of the system consisting of a nanotube (nanocrystal) array and a substrate. In $[8,9]$ we showed that the eigenfrequencies of a single nanoobject can be determined from the examined spectrum of the "integrated (array-substrate) system" and from the spectrum of the substrate.

The method of experimental determination of several first eigenfrequencies of nanoobjects is based on the following idea $[8,9]$. The spectrum of eigenfrequencies of the system consisting of an array of nanotubes (nanocrystals) and a substrate is conventionally divided into two parts. One part of the spectrum refers to the spectrum of eigenfrequencies of the nanoobjects. The substrate remains almost motionless as the nanoobjects oscillate at these frequencies. The other part of the spectrum of the "integrated system" is the spectrum of eigenfrequencies close to the eigenfrequencies of the substrate without nanoobjects. At these frequencies, the amplitude of vibrations of nanoobjects is much smaller than the amplitude of substrate oscillations. Such a division of the spectrum of the "integrated system" into two parts corresponding to the substrate and nanoobject spectra is only possible for several first dozens of eigenfrequencies; at high frequencies, the shapes of natural vibrations are even more complicated. The above-listed properties of the system consisting of an array of nanotubes (nanocrystals) and a substrate were revealed in a theoretical study reported in [8,9]. Based on the theoretical data, two modifications of the experimental procedure for determining the eigenfrequencies of nanoobjects can be proposed.

Modification 1. Several first eigenfrequencies of the system consisting of an array of nanotubes (nanocrystals) and a substrate are measured. Eigenfrequencies of an identical substrate without nanoobjects are measured. The two spectra are compared. The frequencies in the spectrum of the "integrated system "which are close to the frequencies of the substrate without nanoobjects are of no interest. The frequencies in the spectrum of the "integrated system" having no matches in the substrate spectrum are the frequencies of the nanoobjects.

Modification 2. The resonant frequencies of the "integrated system" are measured by registering the electromagnetic response of nanoobjects (this is possible because many nanoobjects possess piezoelectric properties). Then, the amplitude of substrate vibrations is measured (this can be done because the substrate is a macroobject). The resonant frequencies at which the amplitude of substrate oscillations equals zero are the eigenfrequencies of the nanoobjects.

In the case of vertically aligned nanotubes, the method developed in $[8,9]$ makes it possible to evaluate the eigenfrequencies corresponding to the first flexural eigenmodes of the nanotube. From these frequencies, the flexural rigidity of the nanotube as a rod can be determined. For determining the flexural rigidity of the nanofilm from which the nanotube has been rolled, one has to know the eigenfrequencies of nanotubes lying on the substrate.

Methods for fabricating various nanoobjects from multilayer semiconductor nanofilms (GaAs, InAs, GeSi, etc.), including nanotubes aligned horizontally on the substrate, were developed in [13-15]. Effective physical properties of such films essentially depend on their structure and on the residual stress. Semiconductor nanofilms and nanostructures fabricated from such nanofilms are of interest in electronics. Such nanostructures cannot be designed without taking into account mechanical stresses acting in these structures, which are responsible to a large extent for the durability and mechanical strength of nanostructures.

The purpose of the present study was to extend the method of determining the eigenfrequencies of nanoobjects $[8,9]$ to the case of nanotubes attached horizontally to the substrate.

1. Analytical Treatment of a Model Problem. We consider a model consisting of a horizontal plate acting as a substrate and $N$ cylindrical shells lying on the plate, which are treated as nanoobjects (Fig. 1). The plate of thickness $H$ occupies the domain $0 \leq x \leq L, 0 \leq z \leq l$. All shells have identical sizes (i.e., length $l$, radius $R$, and thickness $h$ ) and are equidistantly aligned in the $z$ direction with a gap $a=L /(N+1)$. We assume all the shells to be rigidly attached to the plate.

With the use of the tensor calculus, the governing equations of the linear shell theory can be written as [16-18]

$$
\begin{gathered}
\nabla \cdot \boldsymbol{T}+\rho \boldsymbol{F}=\rho \ddot{\boldsymbol{u}}, \quad \nabla \cdot \boldsymbol{M}+\boldsymbol{T}_{\times}+\rho \boldsymbol{L}=0, \\
\boldsymbol{T} \cdot \boldsymbol{a}+\frac{1}{2}(\boldsymbol{M} \cdot \boldsymbol{b}) \boldsymbol{c}={ }^{4} \boldsymbol{A} \cdots \boldsymbol{\varepsilon}, \quad \boldsymbol{M}^{\mathrm{t}}={ }^{4} \boldsymbol{C} \cdots \boldsymbol{\varkappa}, \\
\boldsymbol{\varepsilon}=\frac{1}{2}\left((\nabla \boldsymbol{u}) \cdot \boldsymbol{a}+\boldsymbol{a} \cdot(\nabla \boldsymbol{u})^{\mathrm{t}}\right), \quad \boldsymbol{\varkappa}=(\nabla \boldsymbol{\varphi}) \cdot \boldsymbol{a}+\frac{1}{2}((\nabla \boldsymbol{u}) \cdots \boldsymbol{c}) \boldsymbol{b}, \\
\boldsymbol{\varphi}=-\boldsymbol{n} \times(\nabla \boldsymbol{u}) \cdot \boldsymbol{n}, \quad \boldsymbol{b}=-\nabla \boldsymbol{n}, \quad \boldsymbol{c}=-\boldsymbol{a} \times \boldsymbol{n} .
\end{gathered}
$$




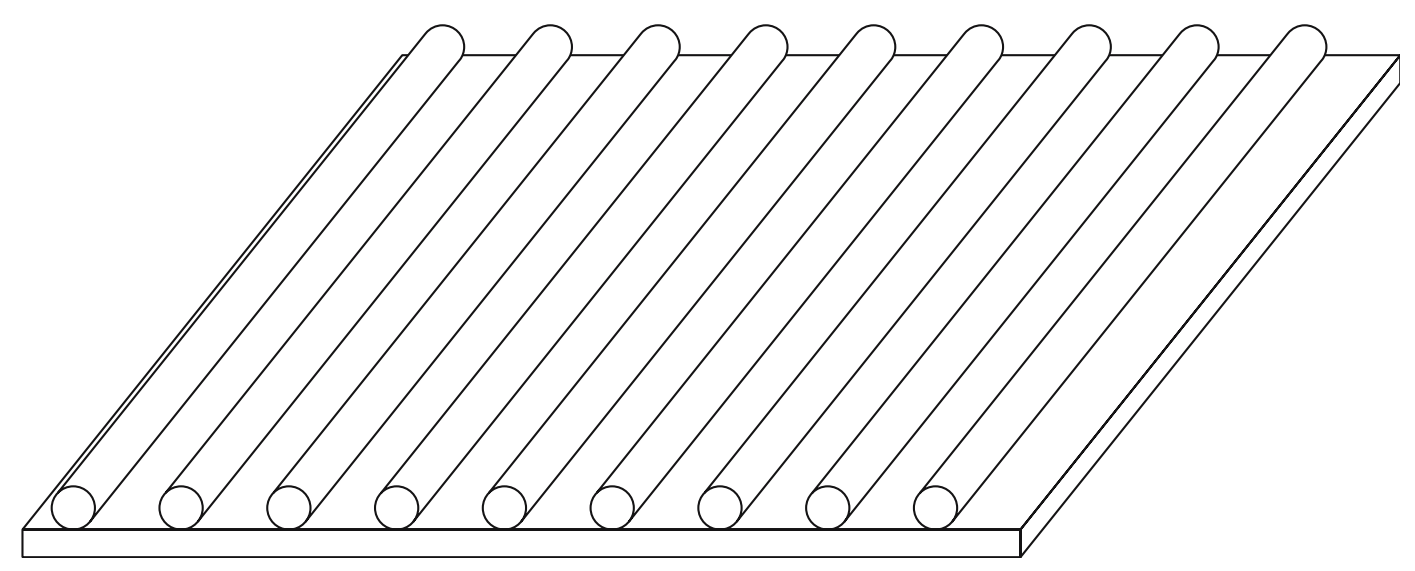

Fig. 1. System of parallel nanotubes lying on a substrate.

Here $\boldsymbol{T}$ and $\boldsymbol{M}$ are the force and moment tensors, $\boldsymbol{T}_{\times}$is the vector invariant of the tensor $\boldsymbol{T}, \rho$ is the surface density, $\boldsymbol{u}$ is the displacement vector, $\boldsymbol{\varphi}$ is the rotation vector, $\boldsymbol{\varepsilon}$ is the tension-shear strain tensor in the tangent plane, $\varkappa$ is the flexure-torsion strain tensor, ${ }^{4} \boldsymbol{A}$ and ${ }^{4} \boldsymbol{C}$ are the rigidity tensors of the shell, $\boldsymbol{n}$ is the unit vector normal to the shell surface, $\boldsymbol{a}$ is the unit vector in the tangent plane, and $\nabla$ is the surface gradient operator.

1.1. Flexural Vibrations of a Cylindrical Shell. To describe the shell kinematics, we use a cylindrical coordinate system $(r, \theta, z)$ with $r \equiv R$. The tension-shear rigidity tensor of the shell in the tangent plane ${ }^{4} \boldsymbol{A}$ is proportional to the shell thickness $h$, and the flexure-torsion rigidity tensor ${ }^{4} \boldsymbol{C}$ is proportional to $h^{3}$. For $h / R \ll 1$ and $h / L \ll 1$, therefore, the shell can be assumed inextensible. Hence, we assume the tension-shear strain tensor to be zero in the tangent plane:

$$
\varepsilon=0
$$

In this case, we have ${ }^{4} \boldsymbol{A} \rightarrow \infty$, the elasticity relation becomes meaningless, and the force tensor in the tangent plane $\boldsymbol{T} \cdot \boldsymbol{a}$ is determined directly from the dynamics equations with regard for the equation of strain compatibility

$$
\Delta(\operatorname{tr}(\boldsymbol{T} \cdot \boldsymbol{a}))-(1+\nu) \nabla \cdot(\nabla \cdot(\boldsymbol{T} \cdot \boldsymbol{a}))=0
$$

( $\nu$ is Poisson's ratio) The flexure-torsion rigidity tensor ${ }^{4} \boldsymbol{C}$ is

$$
{ }^{4} \boldsymbol{C}=D\left(\frac{1+\nu}{2} \boldsymbol{c c}+\frac{1-\nu}{2}\left(\boldsymbol{a}_{2} \boldsymbol{a}_{2}+\boldsymbol{a}_{4} \boldsymbol{a}_{4}\right)\right) .
$$

Here $D$ is the flexural rigidity of the shell, $\boldsymbol{a}_{2}=\boldsymbol{e}_{\theta} \boldsymbol{e}_{\theta}-\boldsymbol{e}_{z} \boldsymbol{e}_{z}$, and $\boldsymbol{a}_{4}=\boldsymbol{e}_{\theta} \boldsymbol{e}_{z}+\boldsymbol{e}_{z} \boldsymbol{e}_{\theta}$.

The displacement and rotation vectors can be decomposed with respect to the basis of the cylindrical coordinate system:

$$
\boldsymbol{u}=u_{\theta} \boldsymbol{e}_{\theta}+u_{z} \boldsymbol{k}+u_{r} \boldsymbol{n}, \quad \boldsymbol{\varphi}=\varphi_{\theta} \boldsymbol{e}_{\theta}+\varphi_{z} \boldsymbol{k} .
$$

Apparently, in the absence of tension-shear strains, all quantities that characterize the stress-strain state of the shell depend only on the polar angle $\theta$. Moreover, Eq. (2) leads to the following kinematic relations:

$$
\frac{d u_{\theta}}{d \theta}+u_{r}=0, \quad u_{z}=0, \quad \varphi_{\theta}=0, \quad \varphi_{z}=\frac{1}{R}\left(u_{\theta}-\frac{d u_{r}}{d \theta}\right) .
$$

The main variable is chosen to be the displacement along the normal to the shell surface $u_{r}$. It can be easily shown that the problem of free oscillations of the shell (1) in the absence of tension-shear strains reduces to the differential equation

$$
\frac{D}{\rho R^{4}} \frac{d^{2}}{d \theta^{2}}\left(\frac{d^{2}}{d \theta^{2}}+1\right)^{2} u_{r}+\left(\frac{d^{2}}{d \theta^{2}}-1\right) \ddot{u}_{r}=0 .
$$

The solutions of Eq. (4) have the following structure:

$$
u_{r}(\theta, t)=U_{r}(\theta) \mathrm{e}^{i \omega t}, \quad U_{r}(\theta)=\sum_{j=1}^{3}\left[A_{j} \sin \left(\lambda_{j} \theta\right)+B_{j} \cos \left(\lambda_{j} \theta\right)\right]
$$


Here $A_{j}$ and $B_{j}$ are arbitrary constants and $\lambda_{j}$ are the roots of the characteristic equation

$$
\lambda^{6}-2 \lambda^{4}+\left(1-\Omega^{2}\right) \lambda^{2}-\Omega^{2}=0,
$$

where $\Omega=\omega \sqrt{\rho / D} R^{2}$ is the dimensionless eigenfrequency whose determination requires the boundary conditions to be formulated.

According to Eq. (5), the function $U_{r}(\theta)$ involves six constants. These constants are determined from the boundary conditions, which are the conditions of periodicity

$$
u_{\theta}(0, t)=u_{\theta}(2 \pi, t), \quad u_{r}(0, t)=u_{r}(2 \pi, t), \quad \varphi_{z}(0, t)=\varphi_{z}(2 \pi, t),
$$

and from the shell-substrate matching conditions formulated below.

1.2. Flexural Vibrations of the Plate. The equations of motion for the plate are

$$
\nabla \cdot \boldsymbol{T}+\sum_{n=1}^{N} \boldsymbol{F}_{n} \delta(x-n a)=\rho_{*} \ddot{\boldsymbol{u}}, \quad \nabla \cdot \boldsymbol{M}+\boldsymbol{T}_{\times}+\sum_{n=1}^{N} \boldsymbol{L}_{n} \delta(x-n a)=0,
$$

where $\delta(x)$ is the Dirac delta-function, $\boldsymbol{F}_{n}$ and $\boldsymbol{L}_{n}$ are the force and the moment acting on the plate from the side of the $n$th cylindrical shell:

$$
\boldsymbol{F}_{n}=\left.\boldsymbol{e}_{\theta} \cdot \boldsymbol{T}^{(n)}\right|_{\theta=0}, \quad \boldsymbol{L}_{n}=\left.\boldsymbol{e}_{\theta} \cdot \boldsymbol{M}^{(n)}\right|_{\theta=0} .
$$

Assuming the plate to be inextensible and ignoring the in-plane shear strain, we bring the equations of motion (7) and (8) to the form

$$
C \Delta \Delta w+\rho_{*} \ddot{w}=-\sum_{n=1}^{N}\left(\left.T_{\theta r}^{(n)}\right|_{\theta=0} \delta(x-n a)+\left.M_{\theta z}^{(n)}\right|_{\theta=0} \delta^{\prime}(x-n a)\right) .
$$

Here $w$ is the plate flexure (displacement in the $y$ direction), and $C$ and $\rho_{*}$ are the flexural rigidity of the plate and its surface density. To close the system, we supplement it with the kinematic conditions of shell matching with the plate

$$
\left.u_{r}^{(n)}\right|_{\theta=0}=-\left.w\right|_{x=n a},\left.\quad u_{\theta}^{(n)}\right|_{\theta=0}=0,\left.\quad \varphi_{z}^{(n)}\right|_{\theta=0}=-\left.\frac{\partial w}{\partial x}\right|_{x=n a}
$$

and with the boundary conditions for the plate

$$
\left.\frac{\partial^{2} w}{\partial z^{2}}\right|_{z=0}=0,\left.\quad \frac{\partial^{2} w}{\partial z^{2}}\right|_{z=l}=0,\left.\quad \frac{\partial^{3} w}{\partial z^{3}}\right|_{z=0}=0,\left.\quad \frac{\partial^{3} w}{\partial z^{3}}\right|_{z=l}=0
$$

in the case of free plate edges at $z=0, l$ and

$$
\left.w\right|_{x=0}=0,\left.\quad w\right|_{x=L}=0,\left.\quad \frac{\partial w}{\partial x}\right|_{x=0}=0,\left.\quad \frac{\partial w}{\partial x}\right|_{x=L}=0
$$

in the case of clamped edges at $x=0, L$.

As we consider the free oscillations of the system, we seek for the solution of Eq. (9) in the form

$$
w(x, z, t)=W(x, z) \mathrm{e}^{i \omega t} .
$$

Substituting the expression for flexure (13) and the expressions for the forces and moments

$$
\left.T_{\theta r}^{(n)}\right|_{\theta=0}=2 R \rho \omega^{2} \sum_{j=1}^{3} \frac{A_{j}^{(n)}}{\lambda_{j}\left(\lambda_{j}^{2}-1\right)},\left.\quad M_{\theta z}^{(n)}\right|_{\theta=0}=\frac{D}{R^{2}} \sum_{j=1}^{3}\left(\lambda_{j}^{2}-1\right) B_{j}^{(n)}
$$

obtained by integrating the equations of motion of the shells into Eq. (9), we obtain the equation

$$
C \Delta \Delta W-\rho_{*} \omega^{2} W=-\sum_{n=1}^{N} \sum_{j=1}^{3}\left(\frac{2 R \rho \omega^{2}}{\lambda_{j}\left(\lambda_{j}^{2}-1\right)} A_{j}^{(n)} \delta(x-n a)+\frac{D\left(\lambda_{j}^{2}-1\right)}{R^{2}} B_{j}^{(n)} \delta^{\prime}(x-n a)\right) .
$$

It should be noted that all strains of the plate dependent on the $z$ coordinate induce tension-compression strains in the shells attached to the plate. In the context of the present study, these vibrations of the shells are of no interest. Below, we consider the plate motions with all quantities characterizing its stress-strain state depending on the $x$ coordinate only. Such motions are admitted by the differential equation (14) and by the boundary 
conditions (11). Obviously, with the condition $l \ll L$ satisfied, several first natural frequencies of plate oscillations are caused by strains depending on the $x$ coordinate only. Hence, instead of Eq. (14), we consider a simpler equation

$$
C W_{x}^{\mathrm{IV}}-\rho_{*} \omega^{2} W=-\sum_{n=1}^{N} \sum_{j=1}^{3}\left(\frac{2 R \rho \omega^{2}}{\lambda_{j}\left(\lambda_{j}^{2}-1\right)} A_{j}^{(n)} \delta(x-n a)+\frac{D\left(\lambda_{j}^{2}-1\right)}{R^{2}} B_{j}^{(n)} \delta^{\prime}(x-n a)\right) .
$$

To proceed with further analysis, we have to determine now the constants $A_{j}^{(n)}$ and $B_{j}^{(n)}(n=1, \ldots, N)$. According to Eqs. (3), (5), (6), and (10), the systems of equations from which these constants can be determined have the form

$$
\begin{gathered}
\sum_{j=1}^{3}\left[A_{j}^{(n)} \sin \left(2 \pi \lambda_{j}\right)-B_{j}^{(n)}\left(1-\cos \left(2 \pi \lambda_{j}\right)\right)\right]=0, \quad \sum_{j=1}^{3} B_{j}^{(n)}=-\left.W\right|_{x=n a}, \\
\sum_{j=1}^{3} \frac{1}{\lambda_{j}} A_{j}^{(n)}=0, \quad \sum_{j=1}^{3} \frac{1}{\lambda_{j}}\left[A_{j}^{(n)} \cos \left(2 \pi \lambda_{j}\right)-B_{j}^{(n)} \sin \left(2 \pi \lambda_{j}\right)\right]=0, \\
\sum_{j=1}^{3} \lambda_{j}\left[A_{j}^{(n)}\left(1-\cos \left(2 \pi \lambda_{j}\right)\right)+B_{j}^{(n)} \sin \left(2 \pi \lambda_{j}\right)\right]=0, \quad \frac{1}{R} \sum_{j=1}^{3} \lambda_{j} A_{j}^{(n)}=\left.W_{x}^{\prime}\right|_{x=n a} .
\end{gathered}
$$

As all the cylindrical shells are assumed to be identical, the determinants of all $N$ systems (16) are also identical. It is only the right sides of systems (16) that differ from each other, because they involve displacements and derivatives of displacements at different points of the plate. Let us consider two cases.

1. The determinant of systems (16) equals zero. In this case, systems (16) have solutions only if their right sides vanish:

$$
\left.W\right|_{x=n a}=0,\left.\quad W_{x}^{\prime}\right|_{x=n a}=0
$$

As the size of the shells modeling the nanoobjects is much smaller than the size of the plate modeling the substrate and the number of shells is sufficiently large, the shells can be assumed to be continuously distributed over the plate surface. Then the discrete conditions (17) can be replaced by the continuous conditions

$$
W(x) \equiv 0, \quad W^{\prime}(x) \equiv 0 .
$$

With conditions (18) satisfied, the plate remains motionless. The vibration frequencies determined from the condition of the zero determinant of systems (16) correspond to oscillations of cylindrical shells lying on a rigid base.

Thus, a spectrum of eigenfrequencies of nanoobject vibrations can be identified within the spectrum of the "integrated system." At these frequencies, the substrate remains motionless.

2. The determinant of systems (16) is other than zero. In this case, systems (16) have unique solutions. The structure of these solutions is such that all constants $A_{j}^{(n)}$ and $B_{j}^{(n)}$ are linear combinations of the quantities $\left.W\right|_{x=n a}$ and $\left.W_{x}^{\prime}\right|_{x=n a}$. It can easily be shown that

$$
\begin{aligned}
& \sum_{j=1}^{3} \frac{2 A_{j}^{(n)}}{\lambda_{j}\left(\lambda_{j}^{2}-1\right)}=\left.G_{1}(\Omega) W\right|_{x=n a}+\left.G_{2}(\Omega) R W_{x}^{\prime}\right|_{x=n a}, \\
& \sum_{j=1}^{3}\left(\lambda_{j}^{2}-1\right) B_{j}^{(n)}=\left.G_{3}(\Omega) W\right|_{x=n a}+\left.G_{4}(\Omega) R W_{x}^{\prime}\right|_{x=n a} .
\end{aligned}
$$

With allowance for Eq. (19), Eq. (15) becomes

$$
C W_{x}^{\mathrm{IV}}-\rho_{*} \omega^{2} W=-\sum_{n=1}^{N}\left[\rho \omega^{2}\left(G_{1} R W+G_{2} R^{2} W_{x}^{\prime}\right) \delta(x-n a)+D\left(\frac{G_{3}}{R^{2}} W+\frac{G_{4}}{R} W_{x}^{\prime}\right) \delta^{\prime}(x-n a)\right] .
$$

If the number of cylindrical shells is sufficiently high, the shells can be assumed to be continuously distributed over the plate surface. By averaging the right side of Eq. (20), we can simplify the mathematical formulation of the problem and reduce it to the equation 


$$
W_{x}^{\mathrm{IV}}-\frac{N D}{C L R^{2}}\left(G_{3} W_{x}^{\prime}+G_{4} R W_{x}^{\prime \prime}\right)-\omega^{2} \frac{\rho_{*}}{C}\left(W-\frac{N R \rho}{L \rho_{*}}\left(G_{1} W+G_{2} R W_{x}^{\prime}\right)\right)=0 .
$$

If the terms due to the presence of the cylindrical shells have small values, the eigenfrequencies of the "integrated system" are close to the eigenfrequencies of the plate without shells. In order of magnitude, these terms can be estimated as

$$
\begin{gathered}
\frac{N D}{C L R^{2}} G_{3} W_{x}^{\prime} \sim N\left(\frac{h}{H}\right)^{3}\left(\frac{L}{R}\right)^{2} W_{x}^{\mathrm{IV}}, \quad \frac{N D}{C L R} G_{4} W_{x}^{\prime \prime} \sim N\left(\frac{h}{H}\right)^{3} \frac{L}{R} W_{x}^{\mathrm{IV}}, \\
\frac{N R \rho}{L \rho_{*}} G_{1} W \sim N \frac{h}{H} \frac{R}{L} W, \quad \frac{N R^{2} \rho}{L \rho_{*}} G_{2} W_{x}^{\prime} \sim N \frac{h}{H}\left(\frac{R}{L}\right)^{2} W .
\end{gathered}
$$

Estimates (22) show that the small value of the dynamic terms due to the presence of the shells is defined exclusively by the small size of the shells in comparison with the plate size. For the force factors due to the presence of the shells to be small, the shell thickness should be much smaller than the plate thickness, and the linear size of the plate and that of the shells should be less different. In fact, the governing factor here is the low value of the quantity $N(h / H)^{3}(L / R)^{2}$.

Two substantial distinctions between the behavior of systems with horizontally and vertically aligned nanotubes deserve mentioning [8]. In the case of vertically aligned nanotubes [8], the equation similar to Eq. (21) contains only even derivatives with respect to the spatial coordinates; in addition, in the case of vibrations with frequencies close to the eigenfrequencies of substrate oscillations, the amplitudes of nanoobject vibrations turn out to be much smaller than those of the substrate. This is not the case for horizontally aligned nanotubes. Physically, this difference arises because the horizontally attached nanotubes in the present work change the effective rigidity of the plate, in contrast to [8] where the vertically aligned distributed nanotubes were assumed to exert no effect on the effective flexural rigidity of the plate. Thus, a plate with horizontally aligned nanotubes, which possesses such effective properties, is anisotropic and inhomogeneous.

2. Numerical Analysis of Natural Vibrations. An analytical study of the spectrum of vibrations of the "integrated system" would be hardly possible without the assumptions made above for the strain state of the system of interest. We use the three-dimensional theory and the finite-element method to study a system of several parallel nanotubes lying on a substrate. As the materials of interest (GaAs, InAs, GeSi, etc.) possess piezoelectric properties $[19,20]$, such a system as a whole can be considered as a composite piezoelectric solid. In the electrostatic approximation and in the absence of mass forces, the governing equations of electroelasticity have the form [21-23]

$$
\begin{gathered}
\rho \ddot{\boldsymbol{u}}=\nabla \cdot \boldsymbol{\sigma}, \quad \nabla \cdot \boldsymbol{D}=0, \\
\boldsymbol{\sigma}=\boldsymbol{C} \cdot \boldsymbol{\varepsilon}-\boldsymbol{e} \cdot \boldsymbol{E}, \quad \boldsymbol{D}=\boldsymbol{e} \cdot \boldsymbol{\varepsilon}+\boldsymbol{\epsilon} \cdot \boldsymbol{E}, \\
\boldsymbol{\varepsilon}=\frac{1}{2}\left(\nabla \boldsymbol{u}+\nabla \boldsymbol{u}^{\mathrm{t}}\right), \quad \boldsymbol{E}=\nabla \varphi,
\end{gathered}
$$

where $\boldsymbol{u}$ is the displacement vector, $\boldsymbol{E}$ is the electric field intensity vector expressed in terms of the vector potential $\varphi$, $\boldsymbol{\sigma}$ is the stress tensor, $\boldsymbol{D}$ is the electric induction vector, $\boldsymbol{\varepsilon}$ is the strain tensor, $\nabla$ is the spatial operator of the gradient, $\rho$ is the density, $\boldsymbol{C}$ is the rigidity matrix, and $\boldsymbol{e}$ and $\boldsymbol{\epsilon}$ are the piezoelectric and dielectric constants.

The boundary conditions for Eqs. (23) are set as follows. Let the body surface $\Gamma$ consist of two parts, $\Gamma=\Gamma_{1} \cup \Gamma_{2}$ and $\Gamma_{1} \cap \Gamma_{2}=\varnothing$. The displacements $\boldsymbol{u}_{0}$ are set on the boundary $\Gamma_{1}$ and the forces $\boldsymbol{f}$ are set on the boundary $\Gamma_{2}$. The boundary conditions are defined by the formulas

$$
\left.\boldsymbol{u}\right|_{\Gamma_{1}}=u_{0},\left.\quad \boldsymbol{n} \cdot \boldsymbol{\sigma}\right|_{\Gamma_{2}}=\boldsymbol{f}
$$

The mechanical boundary conditions (24) are supplemented by the boundary conditions associated with electrical properties. Let $\Gamma=\Gamma_{3} \cup \Gamma_{4}\left(\Gamma_{3} \cap \Gamma_{4}=\varnothing\right)$, with the electrical potential $\varphi_{0}$ set on $\Gamma_{3}$ and the surface charge $q$ set on $\Gamma_{4}$. Then, we obtain

$$
\left.\varphi\right|_{\Gamma_{3}}=\varphi_{0},\left.\quad \boldsymbol{n} \cdot \boldsymbol{D}\right|_{\Gamma_{4}}=q
$$

For the natural vibrations to be analyzed, a solution of the homogeneous boundary-value problem (23)-(25) is sought (at $\boldsymbol{f}=0$ and $q=0$ ) in the form $\boldsymbol{u}=\boldsymbol{U} \mathrm{e}^{i \omega t}$, etc. 


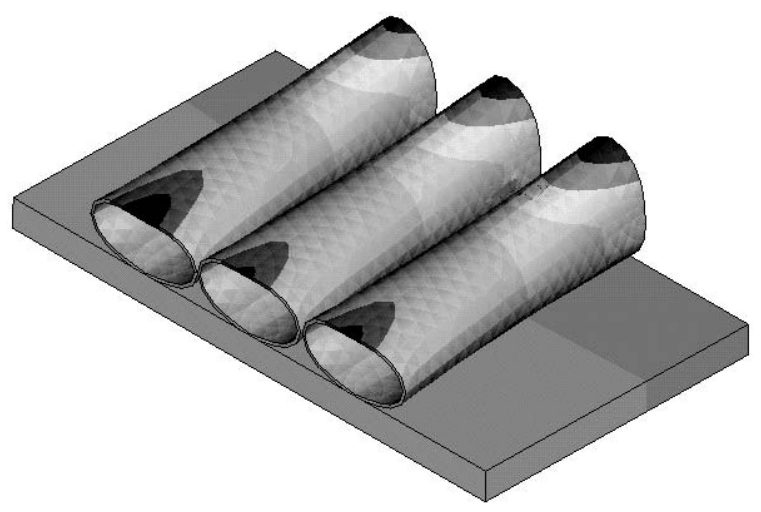

Fig. 2

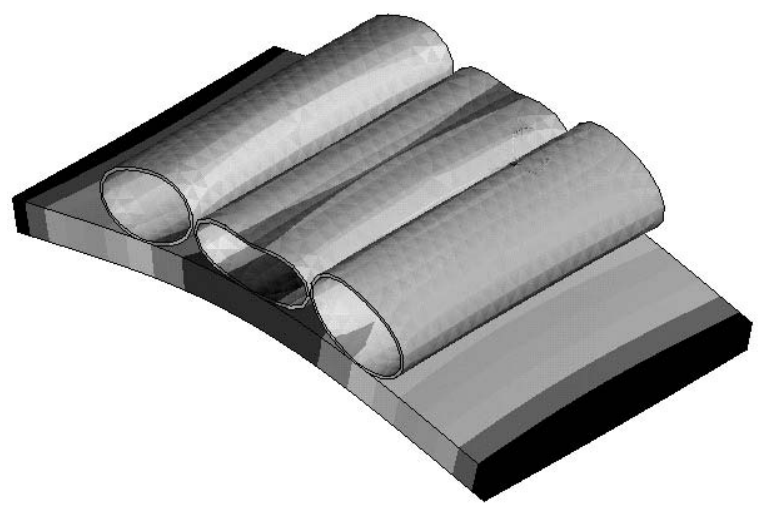

Fig. 3

Fig. 2. Eigenmodes localized in nanotubes.

Fig. 3. Eigenmode corresponding to the first flexural mode of the substrate.

The modal analysis of a three-dimensional system consisting of a plate and parallel nanotubes attached to this plate was performed with the ANSYS software package.

A series of computational experiments was performed with the number of nanotubes varied from one to ten and different geometries of the system (substrate-to-nanofilm thickness ratio, nanotube radius-to-substrate length ratio, etc.). Calculations for different types of substrate fixation were also performed. All components of the system of interest were modeled with linearly elastic three-dimensional anisotropic solids. For the tubes, finite elements corresponding to a piezoelastic material were employed. The calculations were performed for materials commonly used in fabrication of substrates and nanotubes [13-15]. The properties of the materials were borrowed from [19, 20]. The calculations showed that, irrespective of the type of substrate fixation, the spectrum of the "integrated system" displayed the same behavior; namely, the problem parameters could be chosen such that the eigenfrequencies of the nanotubes and those of the substrate could be identified in the overall spectrum. The latter finding provides numerical support to the theoretical analysis performed above.

Some results calculated for a free substrate with three nanotubes are presented below. The substrate was a sapphire crystal, and the physical characteristics of gallium arsenide were adopted for the nanotubes. The geometric parameters of nanotubes were taken from $[13,14]$.

Some eigenmodes of vibrations are shown in Figs. 2-5. Figure 6 shows the distribution of eigenfrequencies in the order of their numbers. For the chosen values of parameters, only three modes of natural vibrations of the substrate were found to be within the frequency range under consideration. Figure 2 shows the eigenmodes that correspond to natural vibrations localized in the nanotubes. The eigenfrequencies of these vibrations correspond to the first eigenfrequency of the nanotube attached to the substrate with a certain portion of its side surface. These frequencies correspond to several first points in Fig. 6. The substrate is seen to be almost motionless in such modes, whereas the nanotubes perform oscillations making the cross-sectional shape of the nanotubes elliptical. Thus, these first frequencies allow the flexural rigidity of the film forming the nanotubes to be evaluated.

Figure 3 shows the eigenmode of vibrations of the nanotube corresponding to the first flexural mode of substrate vibrations. In contrast to the data of $[8,9]$, where the vertically aligned nanoobjects with such modes moved almost as absolutely rigid solids, this mode of vibrations in the case considered corresponds to nanotube deformation, which is fairly natural if the character of nanotube attachment and their lower rigidity are taken into account.

The next modes of natural vibrations refer to a more complex pattern of motion. Figure 4 shows the mode in which the substrate oscillates at the third eigenfrequency, while the nanotubes oscillate at a frequency typical of high-frequency vibrations of a single nanotube. This mode corresponds to the last point in Fig. 6 . Since the nanotubes distributed over the substrate change not only the mass of the system as a whole but also the effective rigidity of the system, it is not only the shape of vibrations that undergoes changes in the case of the modes shown in Fig. 4; in addition, the eigenfrequencies of the "integrated system" differ from the eigenfrequencies of both the 


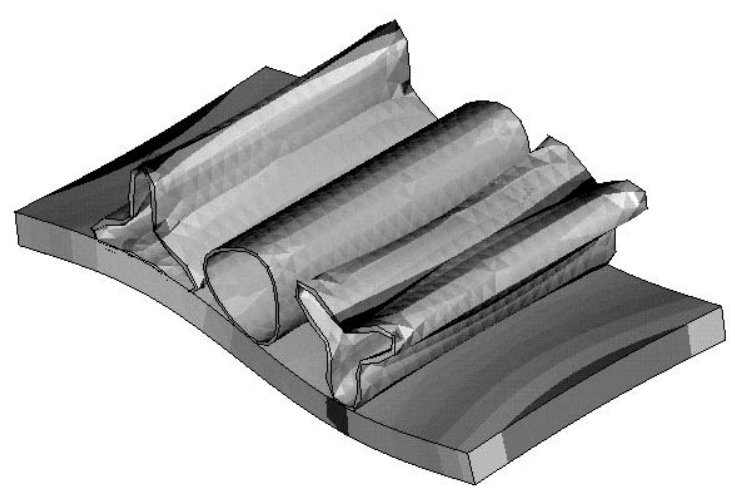

Fig. 4

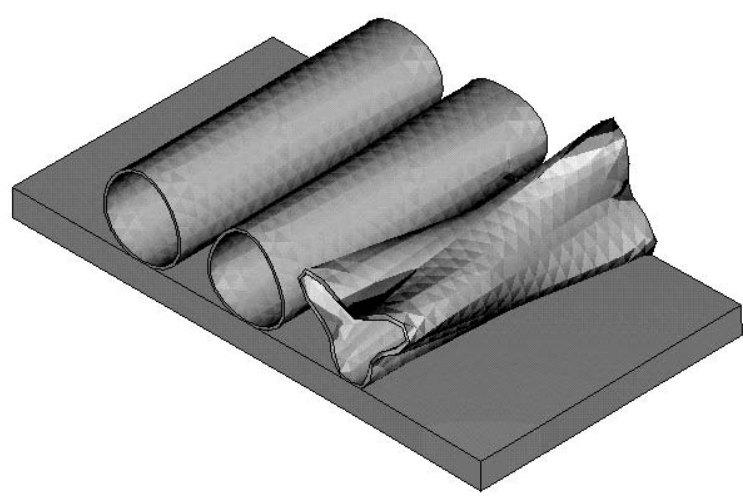

Fig. 5

Fig. 4. High-frequency eigenmode corresponding to integrated substrate and nanotube vibrations.

Fig. 5. Eigenmode localized in a single nanotube.

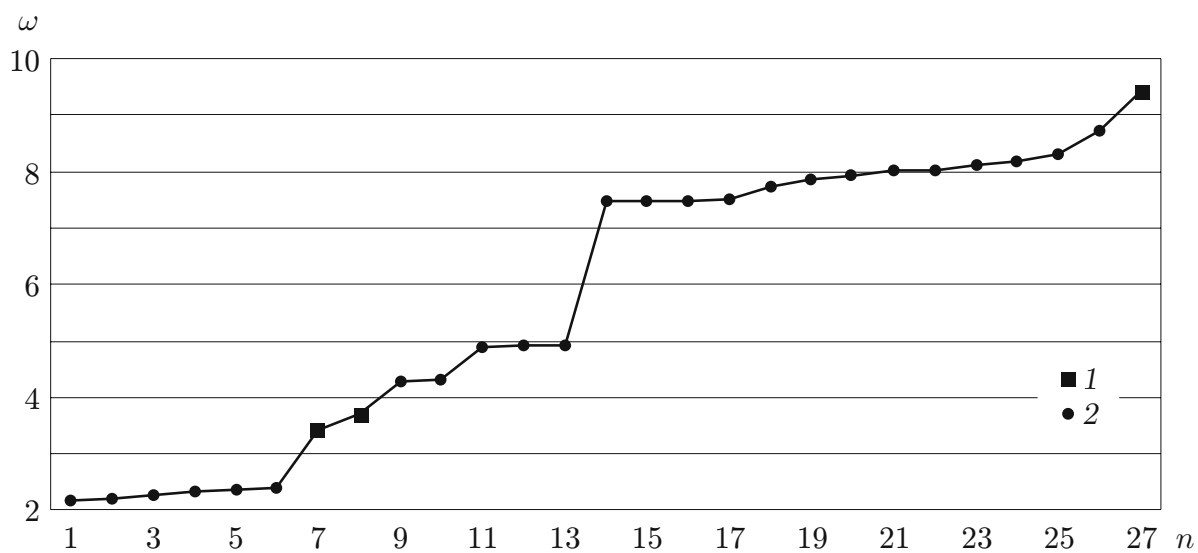

Fig. 6. Eigenfrequencies of the "integrated system" $\omega$ versus their number $n$ : 1 ) eigenfrequencies at which notable substrate motions are observed; 2) eigenfrequencies at which the substrate is almost motionless, while the nanotubes perform oscillations.

substrate and the nanotube. For the first frequencies, the eigenfrequencies of the "integrated system" agree well with the corresponding partial eigenfrequencies of the substrate and the single nanotube.

A numerical analysis also predicts the presence of natural vibrations localized predominantly in one nanotube (Fig. 5). It is worth noting that such vibrations are less interesting from the viewpoint of their experimental detection. In this respect, of greater interest are the localized modes of vibrations in which all nanotubes or the majority of nanotubes oscillate, especially in the case of measurement of electric fields excited in the vicinity of the system (the field strength is higher in the case of a system of oscillating nanotubes). Hence, the effectiveness of the proposed method increases with increasing number of nanotubes on the substrate.

Thus, like in $[8,9]$, we managed to choose geometric parameters of the "integrated system," such that the overall spectrum could be divided into eigenfrequencies caused by natural vibrations of nanotubes and eigenfrequencies of the substrate. In contrast to the vertical array of nanocrystals considered previously, the eigenfrequencies of the substrate and nanotubes here are integrated to a greater extent. The possibility of identifying several first eigenfrequencies of the nanotube from the overall spectrum depends more on the system geometry and its mechanical properties. Thus, in the problem under consideration, computer modeling enables optimization of the experimental scheme to be used in actual tests.

Like the data in Sec. 1, the results of the present numerical analysis allow us to conclude that modifications proposed in the introduction make it possible to detect natural vibrations of an "integrated system" consisting of a substrate and piezoactive nanotubes lying on this substrate. 
3. Conclusions. A method for experimental determination of the flexural rigidity of nanoshells is developed. This method comprises the following steps: 1) excitation of nanoshell vibrations with the shell strain being due to material bending only, the tension and shear being zero or negligibly small; 2) measurement of the first eigenfrequencies of nanoshell oscillations; 3) calculation of the flexural rigidity of the nanoshell from its eigenfrequencies. The main difficulty in practical implementation of the method is the measurement of the eigenfrequency of a single nanoobject.

A method is proposed which enables determination of the first eigenfrequencies of a single nanotube through comparisons of the spectrum of eigenfrequencies of a system consisting of an array of nanoobjects and a substrate with the spectrum of the substrate. The method proved to be most effective and accurate if the first eigenfrequencies of the nanoobjects are comparable with the first eigenfrequencies of the substrate. Thus, the governing factor in using this method is a good choice of proportions between the geometric and physical characteristics of the nanoobjects and the substrate.

The main restriction on application of the method is the frequency range of measuring instruments. If the eigenfrequencies of nanoobjects are too high, such frequencies cannot be registered. It should be noted simultaneously that identical semiconductor nanofilms can be used to create structures of different shapes, such as tubes or cylindrical helical shells [13]. Apparently, such structures have identical flexural rigidities, and the eigenfrequencies of helical shells are notably lower than the eigenfrequencies of tubes [24]. The relation between the eigenfrequencies of helical shells and their length is the same as in the case of rods; hence, by increasing the length of helical shells, one can shift their eigenfrequencies to the range of working frequencies of measuring instruments.

This work was supported by the Russian Foundation for Basic Research (Grant Nos. 05-01-00094-a and 06-01-00452-a), by the Foundation for Supporting National Science, by the Program of the President of the Russian Federation for Supporting the Leading Scientific Schools (Grant No. NSh-4518.2006.1), and by the Program of the President of the Russian Federation for Supporting Young Scientists (Grant No. MD-4829.2007.1).

\section{REFERENCES}

1. M. Yu. Gutkin and I. A. Ovid'ko, Defects and Plasticity Mechanism in Nanostructured and Noncrystalline Materials [in Russian], Yanus, St. Petersburg (2003).

2. M. Yu. Gutkin and I. A. Ovid'ko, Physical Mechanics of Deformable Nanostructures, Vol. 1, Nanocrystalline Materials [in Russian], Yanus, St. Petersburg (2003).

3. A. M. Krivtsov and N. F. Morozov, "Anomalies in mechanical properties of nano-sized objects," Dokl. Ross. Akad. Nauk, 381, No. 3, 345-347 (2001).

4. B. Bhushan (ed.), Springer Handbook of Nanotechnology, Springer-Verlag, Berlin (2004).

5. W. A. Goddard et al. (eds.), Handbook of Nanoscience, Engineering, and Technology, CRC Press, Boca Raton (2003).

6. R. H. Marshall, I. A. Sokolov, Y. N. Ning, et al., "Photoelectromotive force crystals for interferometric measurement of vibrational response," Meas. Sci. Technol., 7, 1683-1686 (1996).

7. S. Gould, Variational Methods for Eigenvalue Problems. An Introduction to the Weinstein Method of Intermediate Problems, London (1966).

8. V. A. Eremeyev, E. A. Ivanova, N. F. Morozov, and A. N. Solov'ev, "On determining eigenfrequencies of nanoobjects," Dokl. Ross. Akad. Nauk, 406, No. 6, 756-759 (2006).

9. V. A. Eremeyev, E. A. Ivanova, N. F. Morozov, and A. N. Solov'ev, "Procedure for determining eigenfrequencies of ordered arrays of nanoobjects," Zh. Tekh. Fiz., 77, No. 1, 3-8 (2007).

10. M. Lorenz, J. Lenzner, E. M. Kaidashev, et al. "Cathodoluminescence of selected single ZnO nanowires on sapphire," Ann. Physik, 2, No. 1, 39-42 (2004).

11. E. M. Kaidashev, M. Lorenz, H. Wenckstern, et al., "High electron mobility of epitaxial ZnO thin films on c-plane sapphire grown by multi-step pulsed laser deposition," Appl. Phys. Lett., 82, 3901-3903 (2003).

12. M. Lorenz, H. Hochmuth, R. Schmidt-Grund, et al., "Advances of pulsed laser deposition of ZnO thin films," Ann. Physik, 13, No. 1, 59-61 (2004).

13. S. V. Golod, V. Ya. Prinz, V. I. Mashanov, and A. K. Gutakovski, "Fabrication of conducting GeSi/Si microand nanotubes and helical microcoils," Semiconductor Sci. Technol., 16, 181-185 (2001). 
14. V. Ya. Prinz, "A new concept in fabricating building blocks for nanoelectronics and nanomechanics devices," Microelectron. Eng., 69, Nos. 2/4, 466-475 (2003).

15. V. Ya. Prinz and S. V. Golod, "Elastic silicon-film-based nanoshells: formation, properties, and applications," J. Appl. Mech. Tech. Fiz., 47, No. 6, 867-878 (2006).

16. P. A. Zhilin, "Master equations of the non-classical shell theory," in: Tr. Leningrad. Politekh. Inst., No. 386, 29-46 (1982).

17. Kh. Al'tenbakh and P. A. Zhilin, "General theory of simple flexible shells," Usp. Mekh., No. 4, 107-148 (1988).

18. P. A. Zhilin, Applied Mechanics. Fundamentals of the Shell Theory [in Russian], Izd. St.-Peterburg. Univ., St. Petersburg (2006).

19. A. A. Blistanov, V. S. Bondarenko, V. V. Chkalova, et al., Acoustic Crystals: Handbook [in Russian], Nauka, Moscow (1982).

20. A. Dargys and J. Kundrotas, Handbook of Physical Properties of Ge, Si, GaAs, and InP, Sci. and Encyclopedia Publ., Vilnius (1994).

21. G. Mougin, Continuum Mechanics of Electromagnetic Solids, North-Holland, Netherlands (1988).

22. V. Z. Parton and B. A. Kudryavtsev, Electromagnetic Elasticity of Piezoelectric and Electroconductive Solids [in Russian], Nauka, Moscow (1988).

23. W. Nowacki, Electromagnetic Effects in Solids [Russian translation], Mir, Moscow (1986).

24. E. A. Ivanova and N. F. Morozov, "Approach to experimental determination of the flexural rigidity of nanoshells," Dokl. Ross. Akad. Nauk, 400, No. 4, 475-479 (2005). 